Check for updates

Cite this: RSC Adv., 2018, 8, 19659

Received 14th March 2018 Accepted 11th May 2018

DOI: $10.1039 / c 8 r a 02237 b$

rsc.li/rsc-advances

\section{Visible-light-driven photocatalytic degradation of safranin-T dye using functionalized graphene oxide nanosheet (FGS)/ZnO nanocomposites $\dagger$}

\begin{abstract}
Bhavani P. Nenavathu, (D) *a Syam Kandula ${ }^{\text {(D) }}{ }^{\mathrm{b}}$ and Swati Verma (iD) ${ }^{\mathrm{c}}$
Photocatalysts suffer from a lack of separation of photogenerated excitons due to the fast recombination of charge carriers, so a strong synergistic effect exhibited by photocatalysts is promising for effective photocatalysis. Herein, we have synthesized efficient visible light functionalized graphene oxide nanosheet (FGS)/ZnO nanocomposite photocatalysts via a simple and economical approach with large scale production for practical applications. A series of nanocomposites (FGS/ZnO NCs) with different amounts by weight of graphene oxide (GO) have been synthesized via a facile solution route followed by calcination under environmental conditions. The phase, purity and morphological studies of the synthesized FGS/ZnO NCs were carried out using powder X-ray diffraction (XRD) and transmission electron microscopy (TEM). The optical properties were studied using UV-visible diffuse reflectance spectroscopy (DRS) and photoluminescence spectroscopy (PL). XRD results confirm the formation of a pure phase of $\mathrm{ZnO}$ in the FGS/ZnO NCs and TEM results show strongly adhered ZnO NPs on the surface of the FGS. DRS results confirm the extension of light absorption in the visible region while PL results confirm the effective separation of charge carriers in 0.09 wt\% FGS/ZnO NCs. The synthesized photocatalyst efficiently degrades carcinogenic safranin-T dye under visible light illumination which is reported for the first time using FGS/ZnO nanocomposites. Photocatalytic studies confirm the higher photocatalytic activity of $0.09 \mathrm{wt} \% \mathrm{FGS} / \mathrm{ZnO} \mathrm{NCs}$ (about 94.5\%) towards the photodegradation of safranin-T dye in aqueous solution under visible light. The improved photocatalytic activity of 0.09 wt $\%$ FGS/ZnO NCs can be ascribed to the integrative synergistic effects of the enhanced adsorption capacity of safranin- $T$ dye, effective separation of photogenerated excitons and effective interfacial hybridization of FGS and ZnO NCs. The generation of reactive oxygen species was confirmed using terephthalic acid as a probe molecule and a scavenger test was conducted in presence of histidine.
\end{abstract}

\section{Introduction}

In the current decade, photocatalysis using either visible light or solar light has become one of the hot research areas due to alarming levels of global warming caused by various industrial pollutants. ${ }^{1-3}$ Organic dyes and their effluents have become the main sources of water contamination, which generally originate

\footnotetext{
${ }^{a}$ Department of Applied Sciences and Humanities, Indira Gandhi Delhi Technical University for Women, Delhi, 110006, India. E-mail: bhavaniprasadnaik@igdtuw.ac. in; Tel: +91-11-23900275

${ }^{b}$ Advanced Materials Institute for BIN Convergence Technology (BK21 Plus Global Program), Department of BIN Convergence Technology, Chonbuk National University, 567, Baekje-daero, Deokjin-gu, Jeonju-si, Jeollabuk-do, 54896, Republic of Korea

${ }^{c}$ Department of Chemical Engineering, Indian Institute of Technology Kanpur, Kanpur208016, India

$\dagger$ Electronic supplementary information (ESI) available: Photographs of the samples, the effect of FGS concentration on adsorption of safranin-T dye, and reusability studies of $\mathrm{FGS} / \mathrm{ZnO} \mathrm{NCs}$ and pristine ZnO NPs. See DOI: $10.1039 / \mathrm{c} 8 \mathrm{ra02237b}$
}

from many industries such as textile, paper, plastic, etc. Among them, textile industries are significant consumers of various organic dyes and release waste effluents into aqueous streams., ${ }^{\mathbf{4 5}}$ In the catalogue of harmful dyes, azo dyes are commonly used in various industries and their associated colour in wastewater is quite noticeable, and they are proven to be potentially carcinogenic and teratogenic to aquatic life as well as the public. ${ }^{4-6}$ These azo dyes are easily water-soluble and difficult to remove or degrade due to their high solubility, stability and the composition ratio of the aromatic rings in their molecular structure. Most of the azo dyes are known to be largely non-biodegradable in aerobic conditions and they are also photochemically active. ${ }^{6,7}$ Therefore, huge research efforts have been devoted to destroying them efficiently in aqueous systems or dispersion systems using UV and solar light. Photocatalysis using UV active semiconductors is quite difficult due to its limited use of the solar spectrum. Additionally, photocatalysis using visible-light-active heteronanostructured semiconductors has shown remarkable progress due to its ease of operation, feasibility, reproducibility, reliability, commercialization, etc. ${ }^{8-11}$ Photocatalytic degradation 
of organic dyes involves the following steps: (i) energy of a photon greater than or equal to the band gap energy of the semiconductor is applied and electrons in the valence band are excited to the conduction band leaving the same amount of holes in the valence band, (ii) the photogenerated excitons can be trapped by dissolved oxygen in aqueous solution resulting in the formation of reactive oxygen species (ROS), for instance ${ }^{\circ} \mathrm{OH}$, and (iii) the ROS can attack and mineralize the adsorbed organic dye molecules. ${ }^{12-14}$ To date, $\mathrm{ZnO}$ and $\mathrm{TiO}_{2}$ based photocatalysts have been widely studied because of their strong oxidizing power, nontoxic nature, low cost and ease of fabrication and because they are environmentally friendly. ${ }^{12-15}$ But the photocatalytic efficiency of these individual materials remains very low because of the fast recombination of photogenerated excitons in the single phase semiconductor. To overcome this drawback, these semiconductor nanomaterials can be coated, deposited or combined with other metals, semiconductors or carbon based materials. ${ }^{\mathbf{1 4 1 6 - 1 8}}$ These nanocomposites can exhibit better and tunable physicochemical properties as compared to those of their counter parts. In the case of the carbon family, graphene has been paid more interest because of its unique optoelectronic properties and many excellent attributes, such as large specific surface area and high transparency. ${ }^{\mathbf{1 9}, 20}$ Meanwhile, graphene oxide (GO) has been receiving increasing attention because it possesses identical properties to graphene as well as a special surface structure with many hydroxyl and carboxyl groups that help to make various heteronanostructures on its surface. ${ }^{21}$ Recent studies have revealed that the governing factors for improving the efficiency of a photocatalyst include increasing the adsorptivity of organic dyes, extending light absorption in the visible range of the solar spectrum and efficient charge separation and transportation of charge carriers to the surface of the photocatalyst, etc. ${ }^{22,23}$ Therefore, it is believed that the anchoring of well-organized $\mathrm{ZnO}$ NPs on GO nanosheets can effectively utilize the combinative features of $\mathrm{ZnO}$ and GO to attain a photocatalyst with superior performance. Junli et al. have synthesized $\mathrm{ZnO} /$ graphene oxide nanocomposites and explored their use for the photocatalytic degradation of methyl orange and water splitting. ${ }^{24}$ Yang et al. have studied the photocatalytic activity of functionalized graphene oxide nanosheets/ZnO for the photodegradation of rhodamine $6 \mathrm{G}$ under ultraviolet light. ${ }^{25}$ Benxia et al., Zhang et al. and Fatin et al. have explored the photocatalytic activity of $\mathrm{ZnO} /$ graphene oxide ( $\mathrm{ZnO} / \mathrm{GO})$ nanocomposites under visible light for the photodegradation of methylene blue. ${ }^{26-28}$ Xinjuan et al. have demonstrated the photocatalytic performance of ZnO-RGO composites towards the reduction of $\mathrm{Cr}(\mathrm{vI}) .{ }^{29}$ Xueshan et al. have fabricated $\mathrm{ZnO}-\mathrm{RGO}$ nanocomposites and explored their use for the photocatalytic degradation of rhodamine $\mathrm{B}^{30}$ Kamat et al. have studied the interaction between graphene oxide and semiconductors $\left(\mathrm{TiO}_{2}\right.$ and $\left.\mathrm{ZnO}\right)$ as well as graphene oxide and metal ( $\mathrm{Au}$ and $\mathrm{Pt}$ ) nanoparticles and explored their use for catalysis, energy conversion and fuel cells. ${ }^{31}$ Zhang et al. have prepared graphene coated $\mathrm{ZnO}$ and explored its use as a photocatalyst for the degradation of methylene blue dye under UV light. They have proposed the Fermi levels as well as the internal electric field using Kelvin probe force microscopy. ${ }^{32} \mathrm{Li}$ et al. have synthesized graphene quantum dots modified with $\mathrm{ZnO}+\mathrm{Cu}$ heterostructures and explored their use for the photocatalytic degradation of rhodamine $\mathrm{B}$ under UV light. ${ }^{33}$ Tian et al. have synthesized N, S co-doped graphene quantum dot (N, S-GQD)reduced graphene oxide ( $\mathrm{rGO})-\mathrm{TiO}_{2}$ nanotube $\left(\mathrm{TiO}_{2} \mathrm{NT}\right)$ composites and explored their use as visible light photocatalysts for the degradation of methyl orange. ${ }^{34}$ Tian et al. have fabricated a ternary hybrid of meso- $\mathrm{TiO}_{2} / \mathrm{RGO} / \mathrm{CdS}$ and used it as a photocatalyst for the degradation of methylene orange under simulated sunlight. ${ }^{35}$ In addition to these reports, various $\mathrm{ZnO}$ and graphene based composite photocatalysts were employed for the degradation of various organic dyes as well as the conversion of toxic gases to environmentally friendly products. ${ }^{36-43}$

In the present study, a facile, economical and simple chemical approach for the synthesis of FGS/ZnO nanocomposites has been reported. The synthesized FGS/ZnO nanocomposites were explored as photocatalysts for the photodegradation of safranin-T (3,7-diamino-2,8-dimethyl-5-phenyl chloride, $\mathrm{C}_{20} \mathrm{H}_{19} \mathrm{~N}_{4} \mathrm{Cl}, M_{\mathrm{W}}=350.85 \mathrm{~g} \mathrm{~mol}^{-1}$ ) in an aqueous solution under visible light irradiation. Some of the reported photocatalysts are only useful under UV light which may not be applicable for practical real-life applications, while the synthetic process of some of the other reported photocatalysts is more complicated. Moreover, the proposed synthetic method could be useful for the large scale production of photocatalysts for real life practical applications. In addition, the photodegradation of safranin-T has been explored for the first time using an FGS/ ZnO photocatalyst and we found excellent photocatalytic activity towards the photodegradation of safranin-T.

\section{Experimental}

Graphite powder $(<20 \mu \mathrm{m}$, Sigma-Aldrich), sulphuric acid (99.99\%, Rankem), potassium permanganate (99.0\%, Rankem), $\mathrm{H}_{2} \mathrm{O}_{2}(30.0 \%$, Rankem), zinc nitrate hexahydrate (99.5\%, Rankem), aqueous ammonia (30.0\%, Rankem), ammonium bicarbonate $\left(99.0 \%\right.$, Rankem), polyvinylpyrrolidone (PVP, $M_{\mathrm{W}}=$ 58 000, Sigma-Aldrich), sodium hydroxide (99.0\%, Rankem), Lhistidine (99\%, Rankem) and terephthalic acid (99.0\%, Rankem) were used as received. Safranin-T (ST) with dye content $\geq 85 \%$ was obtained from Aladdin chemistry co. Ltd., India. All of the chemical reagents and solvents were used as received without any further purification.

\section{Material synthesis}

The synthesis of FGS/ZnO nanocomposites involves two steps and they are described as follows.

\section{Synthesis of graphene oxide (GO)}

GO was prepared by a well-known modified Hummer's method..$^{44}$ About $2 \mathrm{~g}$ of graphite powder was added to a $250 \mathrm{~mL}$ round bottom flask along with $46 \mathrm{~mL}$ of concentrated sulphuric acid. The round bottom flask was kept in an ice bath and about $6 \mathrm{~g}$ of potassium permanganate was slowly added into the flask for up to $2 \mathrm{~h}$. Then, the flask was heated to $36^{\circ} \mathrm{C}$ for $6 \mathrm{~h}$ in an oil bath with continuous stirring. After that, about $92 \mathrm{~mL}$ of deionized water was added dropwise and the stirring continued 
for a further $15 \mathrm{~min}$. The obtained suspension was transferred into a $5 \mathrm{~L}$ beaker and diluted with about $280 \mathrm{~mL}$ of water followed by the addition of $5 \mathrm{~mL}$ of $30.0 \% \mathrm{H}_{2} \mathrm{O}_{2}$. The resulting bright yellow suspension was neutralized $(\mathrm{pH}=7)$ by washing with deionized water several times. Finally, the obtained neutral suspension was centrifuged and the clear suspension was freeze dried for a few days to obtain the GO.

\section{Synthesis of functionalized graphene oxide nanosheet/ZnO nanocomposites (FGS/ZnO NCs)}

Initially, solution A was prepared by mixing a certain amount of zinc nitrate hexahydrate with ammonium bicarbonate in an aqueous solution, and then $2 \mathrm{~mL}$ of aqueous ammonia was added to it. Simultaneously, solution B was prepared by ultrasonication of various amounts of graphene oxide (e.g. 0.05 wt $\%, 0.09$ wt $\%, 0.10 w t \%, 0.50$ wt $\%$ and 5.00 wt $\%$ ) in deionized water along with about $0.1 \mathrm{~g}$ of PVP. Then, the two solutions were properly mixed and stirred for about $2 \mathrm{~h}$ followed by filtration, and washing was carried out with deionized water and ethanol mixture to remove the unbound $\mathrm{Zn}^{2+}$ ions. The obtained powder samples were dried at $80{ }^{\circ} \mathrm{C}$ overnight, and then calcined at $300{ }^{\circ} \mathrm{C}$ for $30 \mathrm{~min}$ under air to obtain $\mathrm{FGS} / \mathrm{ZnO}$ NCs. Similarly, a pristine ZnO NC without GO was also prepared by following the same method without adding the GO as demonstrated above. Photographs of the samples are shown in Fig. S1. $\dagger$

\section{Characterization of pristine and FGS/ZnO NCs}

$\mathrm{X}$-ray diffraction measurements of the pristine $\mathrm{ZnO}$ and FGS/ ZnO NCs were carried out using a Rigaku D/max-3B X-ray diffractometer operated at $40 \mathrm{kV}$ and graphite monochromatized $\mathrm{Cu} \mathrm{K}_{\alpha}(\lambda=1.54 \AA)$ was used as the radiation source in a wide-angle region from $20^{\circ}$ to $80^{\circ}$ on a $2 \theta$ scale. The morphology of the samples was analysed using a JEOL-1200 Transmission Electron Microscope (TEM) operated at $120 \mathrm{kV}$. For TEM analysis, a few milligrams of sample were uniformly dispersed in an ethanol solution and a drop of the suspension placed on a carbon coated 150 mesh copper grid and dried naturally under air at room temperature. UV-visible absorption spectroscopy of the FGS/ZnO NC suspension was measured using a UV-visible spectrophotometer (Hitachi, UV-4100) in the range of 300-500 $\mathrm{nm}$. The band gaps of the FGS/ZnO NCs were measured using the same UV-visible spectrometer with $\mathrm{BaSO}_{4}$ as the reference material. The emission spectra of the FGS/ZnO NCs were recorded using a Hitachi F-4500 fluorescence spectrophotometer at an excitation wavelength $\left(\lambda_{\text {exc }}\right)$ of $385 \mathrm{~nm}$. The Raman spectra of GO and $0.09 \mathrm{wt} \% \mathrm{FGS} / \mathrm{ZnO}$ NCs were recorded using a Raman microscope (Renishaw) with an excitation energy of $\lambda=514 \mathrm{~nm}$.

\section{Photocatalytic degradation study}

For the photocatalytic studies, $100 \mathrm{~mL}$ of $2.2 \times 10^{-4} \mathrm{M}$ safranin$\mathrm{T}$ aqueous dye solution was prepared in a $250 \mathrm{~mL}$ quartz glass beaker. About $20 \mathrm{mg}$ of pristine $\mathrm{ZnO}$ and various FGS/ZnO NCs were separately added to the above beaker and stirred for about $1 \mathrm{~h}$ in the dark to attain an adsorption equilibrium between the photocatalyst and safranin-T dye. The amount of dye adsorption on the photocatalyst's surface was monitored using a UV-visible spectrometer with a time interval of $20 \mathrm{~min}$. Once it was confirmed that there was no further adsorption of dye on the photocatalyst's surface, the safranin-T dye along with the various photocatalysts contained in the quartz glass beaker was illuminated using a HQ1-400W/D lamp with a $420 \mathrm{~nm}$ cut-off filter in the photocatalysis workstation. The distance between the illumination source and the test solution was maintained at about $15 \mathrm{~cm}$. Also, the test quartz beaker was kept under continuous stirring during visible light illumination and water was circulated throughout the photocatalyst chamber to reduce heat generation that could reduce the temperature effect on photocatalytic activity. In addition, a control test was also carried out without adding any photocatalyst. The kinetic study was carried out by using a UV-visible spectrophotometer by withdrawing about a $5 \mathrm{~mL}$ aliquot from the test solution with a time interval of about $10 \mathrm{~min}$. The absorbance of the suspension was measured at $\lambda_{\max }=518 \mathrm{~nm}$ after separating the dispersed NPs using a centrifuge machine (Cence® TG-16-WS) at $15000 \mathrm{rpm}$ at room temperature for $5 \mathrm{~min}$. The photocatalytic activity of the $\mathrm{FGS} / \mathrm{ZnO} \mathrm{NC}$ photocatalysts was compared with that of the pristine ZnO NPs as well as with the control. In order to study the role of GO in the adsorption of dye, a blank experiment was also performed where the same concentration of safranin-T dye solution was treated with a maximum GO concentration comprising $5 \mathrm{wt} \% \mathrm{FGS} / \mathrm{ZnO}$ NCs in the absence of visible light illumination. All of the photocatalyst experiments were performed in triplicate and the obtained results are presented as a mean and standard deviation of three analyses.

\section{Measurement of ' $\mathrm{OH}$ radicals}

About $15 \mathrm{mg}$ of pristine ZnO NPs and FGS/ZnO NCs were sonicated for about $10 \mathrm{~min}$ in a $3 \mathrm{mM}$ solution of terephthalic acid prepared in a $0.01 \mathrm{M} \mathrm{NaOH}$ solution. The reaction mixture was continuously stirred for about $30 \mathrm{~min}$ to form adducts between $\mathrm{OH}^{*}$ and terephthalic acid. Prior to visible light illumination, emission measurements of the test solution were carried out using a Hitachi F-4500 Fluorescence spectrophotometer at an excitation wavelength $\left(\lambda_{\text {exi }}\right)$ of $315 \mathrm{~nm}$. After that, the test solution was illuminated under visible light and about a $5 \mathrm{~mL}$ aliquot was withdrawn at periodic intervals. After centrifugation, the obtained supernatant was examined using the same instrument. A control experiment was also performed without adding the photocatalyst under identical conditions. The total number of $\mathrm{OH}^{\bullet}$ radicals generated during the course of the reaction was measured using the fluorescence intensity of the adducts formed between $\mathrm{OH}^{\cdot}$ and terephthalic acid. ${ }^{\mathbf{1 4 4 5}}$ All of the analyses were performed in triplicate and the results are represented as a mean and standard deviation of three analyses.

\section{Photocatalytic degradation of safranin-T dye in the presence of histidine}

We have also studied the role of reactive oxygen species (ROS) towards the photocatalytic degradation of aqueous safranin-T 
dye solution using histidine as a ROS scavenger. ${ }^{46,47}$ About $25 \mathrm{~mL}$ test samples comprising the same concentration of safranin-T dye with about $5 \mathrm{mg}$ of $0.09 \mathrm{wt} \% \mathrm{FGS} / \mathrm{ZnO} \mathrm{NC}$ and about $45 \mathrm{mg}$ of histidine were continuously stirred under visible light illumination. The time dependent kinetic study of the test solution was carried out using the same UV-visible spectrophotometer. All of the analyses were performed in triplicate and the obtained results are represented as a mean and standard deviation of three analyses.

\section{Results and discussion}

\section{Characterization of as-synthesized FGS/ZnO NCs}

At first, the phase purity of the pristine ZnO NP and FGS/ZnO (0.09 wt\%) NC samples were analysed using XRD analysis and the corresponding results are shown in Fig. 1. Both of the samples exhibit diffraction peaks at $2 \theta$ values of $31.9^{\circ}, 34.4^{\circ}$, $36.3^{\circ}, 47.6^{\circ}, 56.7^{\circ}, 62.9^{\circ}$ and $68.1^{\circ}$, which correspond to the (100), (002), (101), (102), (110), (103) and (112) planes of the hexagonal wurtzite phase of ZnO (JCPDS card no. 05-0664). The crystallite sizes of the pristine ZnO NPs and FGS/ZnO (0.09 wt\%) NCs were derived using the Debye-Scherrer formula. ${ }^{48}$ The crystallite sizes of the ZnO NPs were calculated using the XRD peaks at $2 \theta=36.3^{\circ}$ and they were found to be about 10.3 and $9.3 \mathrm{~nm}$ for the pristine ZnO NPs and FGS/ZnO (0.09 wt\%) NCs, respectively. However, we haven't observed any diffraction peaks of the functionalized graphene oxide nanosheets, which can be ascribed to the as-synthesized GO nanosheets having either an amorphous nature or a weak crystalline nature. Moreover, the absence of GO diffraction peaks gives indirect evidence for the uniform coverage of $\mathrm{ZnO}$ NPs on the surface of FGS and vice versa. To prove the presence of GO in the FGS/ZnO nanocomposites, we have carried out Raman analysis, as shown in Fig. 1b. The Raman spectra of graphene oxide and $0.09 \mathrm{wt} \%$ FGS/ZnO nanocomposites show characteristic $D$ and $G$ bands at about $1360 \mathrm{~cm}^{-1}$ and $1605 \mathrm{~cm}^{-1}$, respectively. The $I_{\mathrm{D}} / I_{\mathrm{G}}$ ratio was determined to be 1.02 and 1.18 for bare $\mathrm{ZnO}$ and 0.09 wt\% FGS/ZnO nanocomposites, respectively. These results indicate that there is an increase of defects in the case of $0.09 \mathrm{wt} \% \mathrm{FGS} / \mathrm{ZnO}$ nanocomposites, which can effectively trap the charge carriers and reduce the recombination rate. In addition, one more peak at about $495 \mathrm{~cm}^{-1}$ is observed in the case of $0.09 \mathrm{wt} \% \mathrm{FGS} / \mathrm{ZnO}$ nanocomposites, which could be due to an $\mathrm{E}_{2}$ high vibration mode associated with the wurtzite type $\mathrm{ZnO}$.

The morphology of the pristine $\mathrm{ZnO}$ and FGS/ZnO $(0.05 \mathrm{wt} \%, 0.09 \mathrm{wt} \%$ and $5.00 \mathrm{wt} \%)$ NCs was examined using TEM analysis and the corresponding figures are shown in Fig. 2. The pristine ZnO NPs show aggregated nanoparticles with an average size of about 10 to $20 \mathrm{~nm}$ (Fig. 2a). In the case of FGS/ZnO NCs, strongly adhered ZnO NPs on the surface of FGS have been observed, which can be ascribed to strong bonding between the ZnO NPs and the functionalized surface of the GO nanosheets. In the case of the FGS/ZnO NCs, 0.05 wt $\%$ FGS supported $\mathrm{ZnO}$ shows a slight aggregation of ZnO NPs on the surface of FGS (Fig. 2b), while 0.09 wt\% FGS supported ZnO shows better deposition of ZnO NPs with
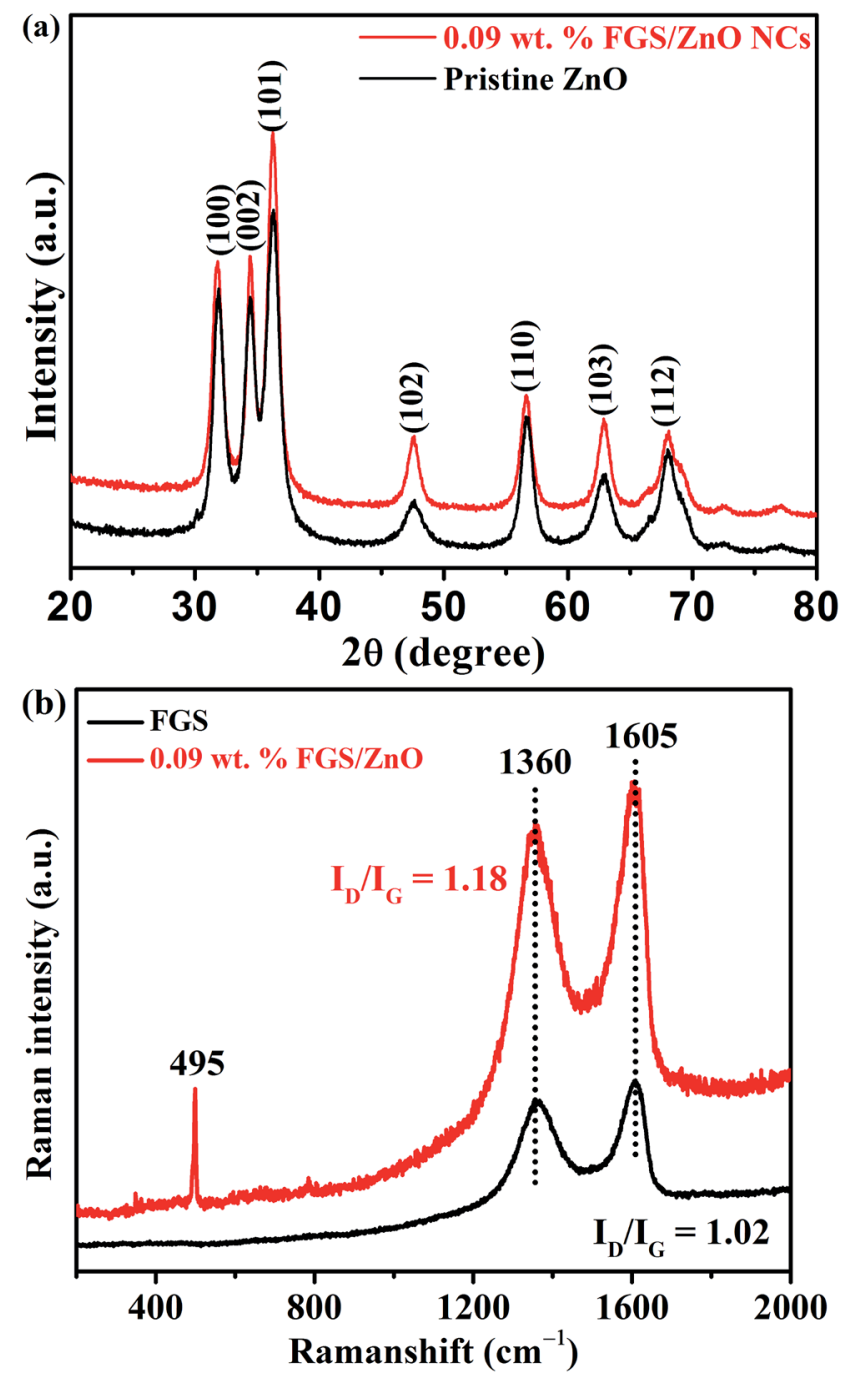

Fig. 1 (a) XRD spectra and (b) Raman spectra of pristine $\mathrm{ZnO}$ and FGS/ $\mathrm{ZnO}(0.09$ wt\%) NCs.

uniform distribution observed (Fig. 2c). 5.00 wt\% FGS supported $\mathrm{ZnO}$ shows more free space with a lesser amount of ZnO NPs on the surface of FGS (Fig. 2d). Fig. 2e clearly shows lattice spacing of about $0.33 \mathrm{~nm}$ and $0.28 \mathrm{~nm}$ due to the (002) plane of graphene and (100) plane of $\mathrm{ZnO}$, respectively. The close lattice spacing between $\mathrm{ZnO}$ and FGS can favour fast carrier transfer and separation. The EDS spectrum confirms the presence of zinc, oxygen and carbon in $0.09 \mathrm{wt} \% \mathrm{FGS} / \mathrm{ZnO}$ nanocomposites and also indicates the uniform distribution of all of the elements in the $0.09 \mathrm{wt} \% \mathrm{FGS} / \mathrm{ZnO}$ nanocomposite (Fig. 2f). These results give strong evidence for the formation of FGS/ZnO nanocomposites. These results suggest that $0.09 \mathrm{wt} \%$ FGS supported ZnO can be a better photocatalyst for the degradation of dye due to the uniform distribution of $\mathrm{ZnO}$ NPs that can efficiently absorb the visible light and effectively separate the charge carriers at the surface of a photocatalyst. Moreover, the disappearance of wrinkles of FGS is observed, which can be ascribed to the uniform decoration of ZnO NPs on exfoliated functionalized GO nanosheets. 

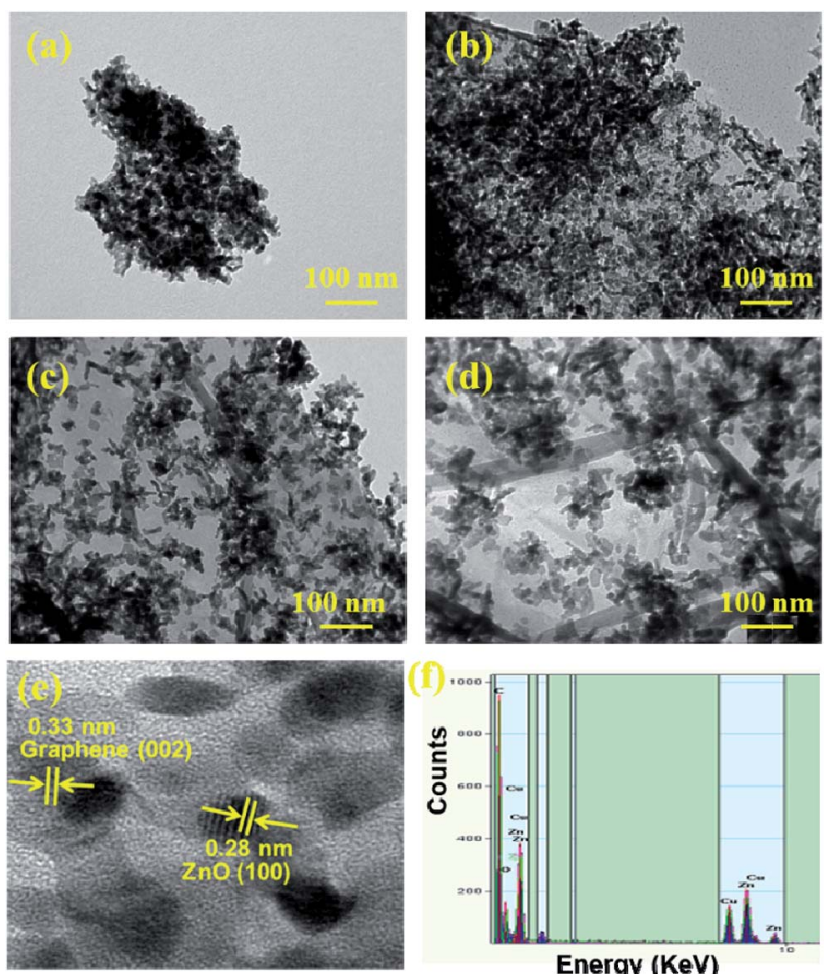

Energy (KeV)

Fig. 2 TEM images of (a) pristine $\mathrm{ZnO}$, (b) 0.05 wt\% FGS/ZnO NCs, (c) 0.09 wt\% FGS/ZnO NCs, (d) 5.00 wt\% FGS/ZnO NCs, (e) HRTEM image, and (f) EDS spectrum of 0.09 wt\% FGS/ZnO NCs.

\section{Optical studies of as-synthesized FGS/ZnO NCs}

The optical properties of pristine ZnO NPs and various compositions of FGS/ZnO NCs were studied using UV-visible spectroscopy and photoluminescence spectroscopy. Initially, the optical band gaps of the samples were studied using UVvisible diffuse reflectance spectroscopy and the corresponding figures are shown in Fig. $3 \mathrm{a}$ and b. The pristine ZnO NPs and various FGS/ZnO NCs showed absorption between 390-420 nm. The band gaps of the samples were measured using Tauc plots (Fig. $3 \mathrm{~b}){ }^{49}$ The pristine ZnO NPs showed a band gap of about $3.16 \mathrm{eV}$, which is blue shifted to that of bulk $\mathrm{ZnO}$ (about 3.38 $\mathrm{eV}$ ), which can be attributed to the smaller size of the ZnO NPs as well as quantum confinement. ${ }^{50,51}$ The FGS/ZnO NCs exhibited band gaps of about 3.13, 3.10, 3.12, 3.18 and $3.27 \mathrm{eV}$ corresponding to $0.05 \mathrm{wt} \%, 0.09 \mathrm{wt} \%, 0.10 \mathrm{wt} \%, 0.50 \mathrm{wt} \%$ and $5.00 \mathrm{wt} \%$ of the FGS/ZnO NCs, respectively. Among the FGS/ZnO NCs, $0.09 \mathrm{wt} \% \mathrm{FGS} / \mathrm{ZnO}$ NCs exhibited a lower band gap and a blue shift of about $0.06 \mathrm{eV}$ as compared to those of pristine ZnO NPs, which can be attributed to the quantum confinement of ZnO NPs on the surface of FGS. ${ }^{50,51}$ In addition, the increased absorbance of the FGS/ZnO NCs $(0.05 \mathrm{wt} \%, 0.09 \mathrm{wt} \%$ and $0.10 \mathrm{wt} \%)$ in the visible region as compared to that of pristine $\mathrm{ZnO}$ indicates the formation of visible light photocatalysts and that they can be effective for practical photocatalytic applications under visible light or sunlight. These band gap results further supported the higher photocatalytic activity of $0.09 \mathrm{wt} \%$ FGS/ZnO NCs in visible light.
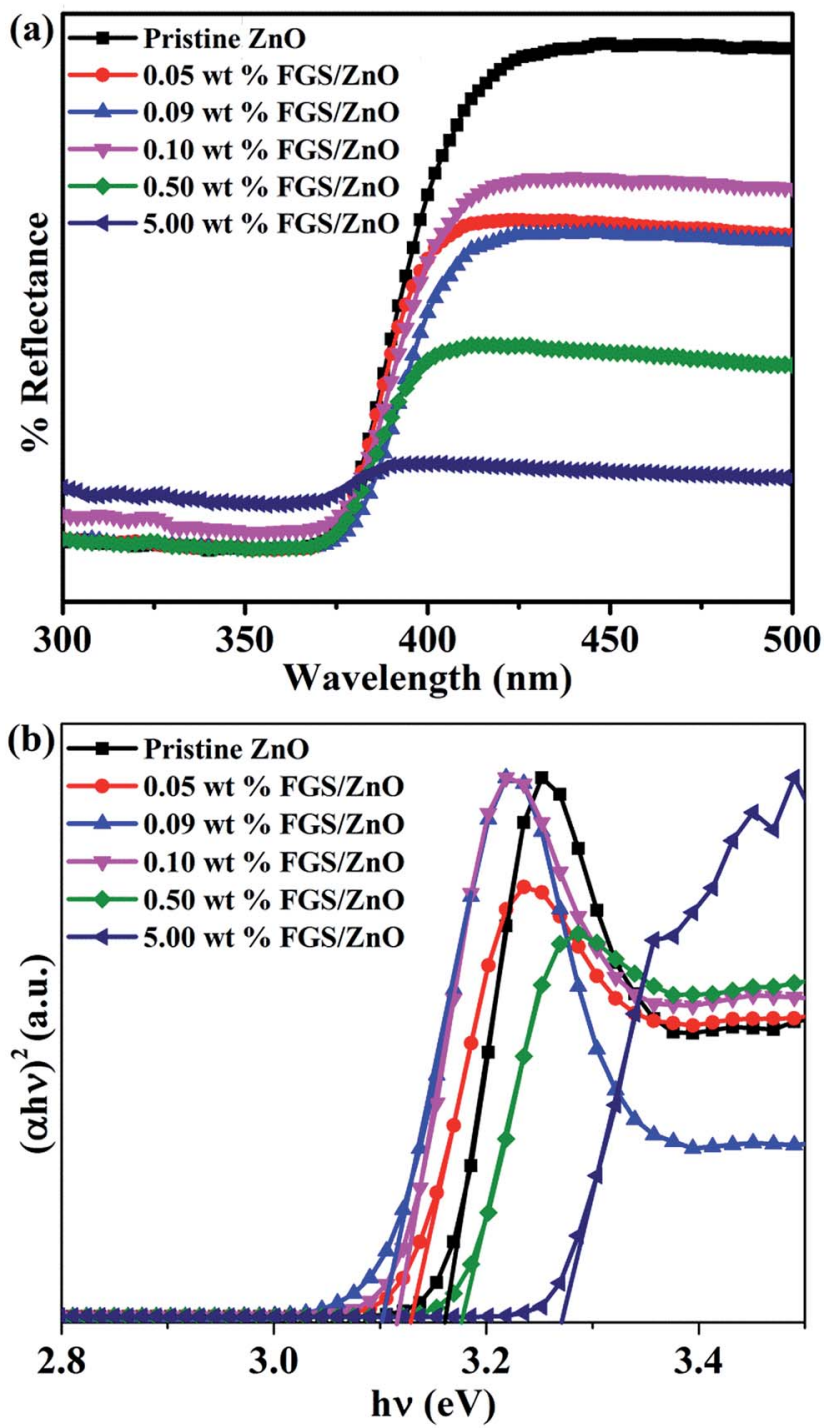

Fig. 3 (a) The UV-visible diffuse reflectance spectra and (b) Tauc plots of pristine ZnO NPs and FGS/ZnO NCs.

The photoluminescence spectra of pristine ZnO NPs and various FGS/ZnO NCs are shown in Fig. 4. The PL spectra of pristine ZnO NPs as well as FGS/ZnO NCs show weak emission bands at $435 \mathrm{~nm}, 466 \mathrm{~nm}$ and $485 \mathrm{~nm}$. The emission band in the blue region at $435 \mathrm{~nm}$ is ascribed to the electronic transition between interstitial oxygen $\left(\mathrm{O}_{\mathrm{i}}\right)$ and the excitonic level. ${ }^{18,52}$ Another emission in the blue-green region of about $466 \mathrm{~nm}$ is ascribed to the electronic transition between a shallow donor $\left(\mathrm{Zn}_{\mathrm{i}}\right)$ and deep acceptor $\left(\mathrm{V}_{\mathrm{Zn}}\right)^{{ }^{14,53}}$ The PL emission in the green region of about $485 \mathrm{~nm}$ is attributed to interstitial zinc, structural defects and zinc vacancies. ${ }^{53}$ In addition, $0.50 \mathrm{wt} \%$ and $5.00 \mathrm{wt} \% \mathrm{FGS} / \mathrm{ZnO}$ NCs exhibit a strong new green emission at about $565 \mathrm{~nm}$ ascribed to structural defects caused by oxygen vacancies. ${ }^{25}$ The green emission peak intensity is increased by increasing the concentration of FGS indicating that there are more structural defects that reduce the photocatalytic activity of the high loaded FGS NCs. Additionally, a decrease in PL emission intensity is observed for $0.09 \mathrm{wt} \% \mathrm{FGS} / \mathrm{ZnO}$ NCs compared 


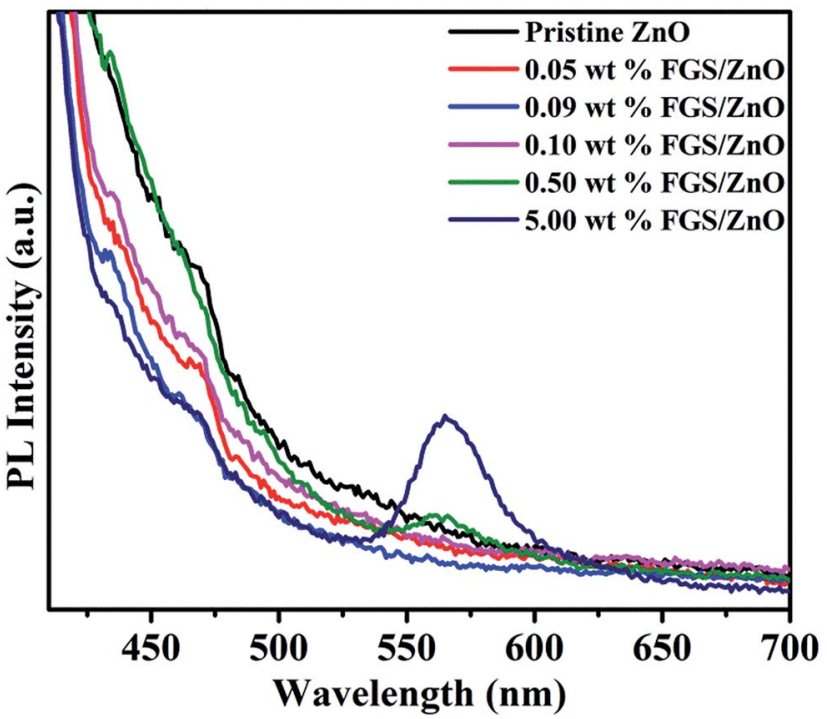

Fig. 4 The photoluminescence (PL) spectra of pristine ZnO NPs and FGS/ZnO NCs.

to that of pristine ZnO NPs and other FGS/ZnO NCs. The likely reason for the reduced PL emission intensity is due to the suppression of exciton recombination by the optimized amount of FGS in the photocatalyst, which is further supported by an increase in the photocatalytic activity and the generation of reactive oxygen species (ROS). The observed results corroborate with the reported literature related to the enhanced photocatalytic activity of $\mathrm{TiO}_{2}$ (B)/anatase core-shell heteronanostructures and photocatalytic reduction of $\mathrm{Cr}(\mathrm{vI})$ using pristine $\mathrm{ZnO}$ NPs and $\mathrm{ZnO} / \mathrm{rGO}$ composites under visible irradiation. ${ }^{29,54}$ All of these results demonstrate that $0.09 \mathrm{wt} \% \mathrm{FGS} /$ ZnO NCs can act as a better photocatalyst for the photodegradation of safranin-T dye.

\section{Photocatalytic studies}

Before visible light illumination on the test solution, we have examined the effect of FGS concentration in FGS/ZnO NCs on adsorption. According to literature, the available functional groups such as hydroxyl, carbonyl and epoxy groups on the surface of the GO nanosheets, and the $\pi-\pi$ conjugation and 2-D planar structure can contribute to the adsorption of dye molecules on its surface..$^{24,25}$ To study the adsorption of dye on the surface of $\mathrm{FGS} / \mathrm{ZnO} \mathrm{NCs}, 20 \mathrm{mg}$ of various photocatalysts were dispersed into $100 \mathrm{~mL}$ of $2.2 \times 10^{-4} \mathrm{M}$ dye solution and stirred for about $7 \mathrm{~h}$ under dark conditions. Interestingly, almost complete adsorption of safranin-T dye onto the surface of the $5.00 \mathrm{wt} \% \mathrm{FGS} / \mathrm{ZnO}$ NCs was observed as compared that adsorbed by the $0.09 \mathrm{wt} \%$ sample, which could be due to the availability of a higher amount of surface functional groups on the surface of FGS for dye adsorption (Fig. S2 $\dagger)^{24,25}$ In this way, the role of FGS on the adsorption of safranin-T dye in the FGS/ZnO NCs was established.

Next, the photocatalytic activity of the pristine ZnO NPs and FGS/ZnO NCs was examined towards the photodegradation of safranin- $T$ dye in an aqueous solution under visible light and the corresponding results are shown in Fig. 5. The observed results indicate that FGS/ZnO NCs (0.05 wt\%, $0.09 \mathrm{wt} \%$ and $0.10 \mathrm{wt} \%)$ possess higher photodegradation efficiency than the pristine $\mathrm{ZnO}$ NPs, FGS/ZnO NCs (0.50 wt\% and $5.00 \mathrm{wt} \%$ ) and control sample (Fig. 5a). The lower photocatalytic activity of $0.50 \mathrm{wt} \%$ and $5.00 \mathrm{wt} \% \mathrm{FGS} / \mathrm{ZnO}$ NCs than that of other FGS/ZnO NCs could be due to a shielding effect. By increasing the concentration of FGS in FGS/ZnO NCs, they are able to shield the active sites on the surface of the photocatalyst and significantly reduce the penetration of light to the active centres. ${ }^{27}$ In addition, the higher concentration of FGS leads to more opacity and light scattering results that show a decrease in the intensity of light penetration to the active centres of the photocatalyst. ${ }^{26}$ Moreover, the increased concentration of FGS in FGS/ZnO NCs can absorb some of the light and cause the problem of light harvesting competition between the counter parts of the FGS/ZnO NC. ${ }^{2}$ This leads to high recombination centres instead of providing an efficient electron pathway and thus reduces the photocatalytic activity.

Kinetic studies were performed for all of the samples and the corresponding results are given in Fig. $5 \mathrm{~b}$ and c. The photodegradation of safranin- $T$ dye follows pseudo-first-order kinetics, which can be written as $^{14}$

$$
\ln C_{\mathrm{o}} / C_{t}=k t
$$

where $C_{\mathrm{o}}$ and $C_{t}$ are the concentrations of safranin-T dye at time $=0$ and time $=t$, respectively, and $k$ is the apparent pseudo-first-order rate constant. The linear relationship between $\ln \left(C_{\mathrm{o}} / C_{t}\right)$ and irradiation time indicates that the photodegradation of safranin-T dye follows pseudo-first-order kinetics. ${ }^{18}$ The apparent pseudo-first-order rate constant $(k)$ was calculated from the slope of the plot of $\ln \left(C_{\mathrm{o}} / C_{t}\right) v s$. time ' $t$ '. The estimated apparent pseudo-first-order rate constant $(k)$ values for the degradation of safranin-T are $0.018,0.026,0.049$, $0.031,0.017$ and $0.014 \mathrm{~min}^{-1}$ for pristine $\mathrm{ZnO}$, and $0.05 \mathrm{wt} \%$,
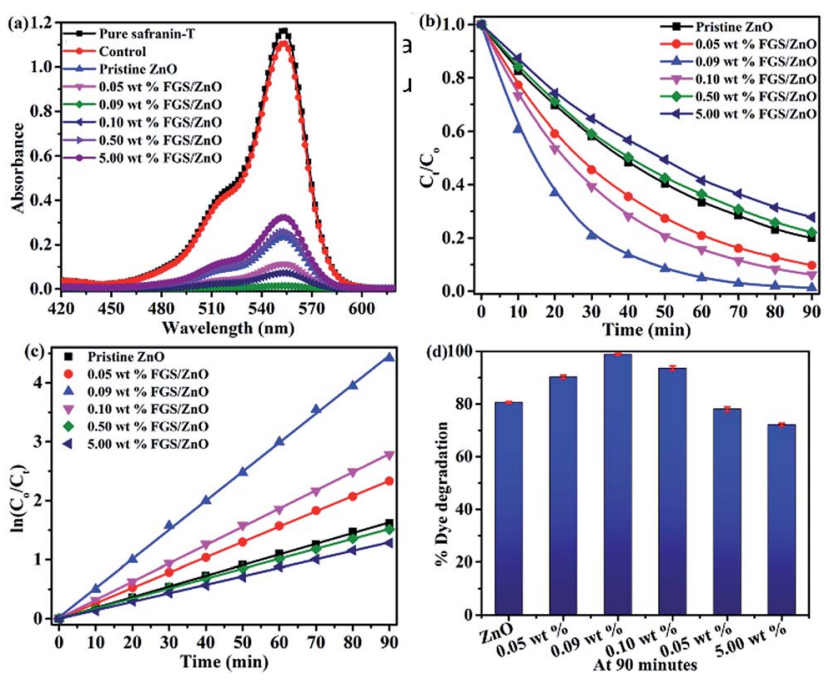

Fig. 5 (a) Photodegradation of safranin-T using pristine $\mathrm{ZnO} N P s$ and FGS/ZnO NCs, (b) comparison of the photocatalytic activity of FGS/ZnO $\mathrm{NCs}$ with that of pristine $\mathrm{ZnO} N P s$, (c) plots of $\ln \left(C_{0} / C_{t}\right)$ vs. irradiation time using different photocatalysts and (d) comparison of safranin- $T$ photodegradation efficiency using ZnO NPs and FGS/ZnO NCs. 
$0.09 \mathrm{wt} \%, 0.10 \mathrm{wt} \%, 0.50 \mathrm{wt} \%$ and $5.00 \mathrm{wt} \%$ FGS in FGS/ZnO NCs, respectively. The order of photodegradation efficiency of the photocatalysts is $0.09 \mathrm{wt} \% \mathrm{FGS} / \mathrm{ZnO} \mathrm{NCs}>0.10 \mathrm{wt} \% \mathrm{FGS} /$ ZnO NCs $>0.05 \mathrm{wt} \% \mathrm{FGS} / \mathrm{ZnO}$ NCs $>$ pristine $\mathrm{ZnO}>0.50 \mathrm{wt} \%$ FGS/ZnO NCs $>5.00 \mathrm{wt} \%$ FGS/ZnO NCs (Fig. 5d). The higher degradation efficiency of $0.09 \mathrm{wt} \% \mathrm{FGS} / \mathrm{ZnO}$ NCs as compared to that of pristine $\mathrm{ZnO}$ and other FGS/ZnO NCs could be due to the effective separation of charge carriers as well as the effective transportation of charge carriers to the surface of the photocatalyst, which can be seen in the PL spectra of the FGS/ZnO NCs in Fig. 4. The decreased photocatalytic activity in the cases of $0.50 \mathrm{wt} \%$ and $5.00 \mathrm{wt} \% \mathrm{FGS} / \mathrm{ZnO}$ NCs could be due to the formation of a higher amount of oxygen vacancies, which is evident from the intense emission band at $565 \mathrm{~nm}$ (Fig. 4). The observed results corroborate well with the reported literature on the photocatalytic degradation of various dyes using $\mathrm{ZnO} / \mathrm{rGO}$ nanocomposites. ${ }^{27}$ The photodegradation efficiency of various $\mathrm{ZnO} /$ graphene or $\mathrm{ZnO} /$ graphene oxide composite photocatalysts are summarized and shown in Table S1. $\uparrow$ The reusability of a photocatalyst elucidates its stability and activity. One of the important problems associated with using ZnO NPs as a photocatalyst is that they have lower photostability and can easily undergo photoinduced dissolution. ${ }^{55}$ The possible photocorrosion reaction can be expressed as follows:

$$
\mathrm{ZnO}+2 \mathrm{~h}^{+} \rightarrow \mathrm{Zn}^{2+}+1 / 2 \mathrm{O}_{2}
$$

The most probable reason for photocorrosion of $\mathrm{ZnO}$ is due to the reaction of holes present in the valence band with surface oxygen at the solid interface of the photocatalyst. To test the photocatalytic stability (reusability) $0.09 \mathrm{wt} \% \mathrm{FGS} / \mathrm{ZnO}$ NCs and pristine $\mathrm{ZnO}$ were chosen and photocatalytic experiments were carried out for up to 5 cycles (Fig. S3 $\dagger$ ). The 0.09 wt $\%$ FGS/ZnO NCs showed about $4 \%$ lower degradation after five cycles while the activity of pristine $\mathrm{ZnO}$ was drastically reduced, indicating good stability and durability of the $0.09 \mathrm{wt} \% \mathrm{FGS} / \mathrm{ZnO}$ NCs over pristine ZnO NPs. These results demonstrate that the combination of optimized FGS and ZnO improves the photostability as well as the photocatalytic activity of the FGS/ZnO NCs.

The proposed possible mechanism for the photodegradation of safranin-T by FGS/ZnO NCs is shown in Fig. 6..$^{24,27,29}$ Upon visible light illumination, electrons $\left(\mathrm{e}^{-}\right)$present in the valence band of $\mathrm{ZnO}$ are excited to the conduction band and leave the same number of photogenerated holes $\left(\mathrm{h}^{+}\right)$in the valence band. Then, the electrons present in the conduction band of $\mathrm{ZnO}$ are able to transfer to the functionalized GO nanosheets due to strong interfacial contact between $\mathrm{ZnO}$ and FGS, which effectively hinders the recombination rate of photoexcited excitons and effectively transports the charge carriers towards the surface of the photocatalyst. The offset face-to-face free safranin-T dye adsorbed over the surface of FGS via $\pi-\pi$ interactions can be directly oxidized by the holes present in the valence band of $\mathrm{ZnO}$. In addition, the electrons present on the surface of the photocatalyst are readily trapped by dissolved oxygen $\left(\mathrm{O}_{2}\right)$ in the aqueous solution and produce superoxide radical anions $\left(\mathrm{O}_{2}{ }^{-}\right)$. The reactive active species such as holes

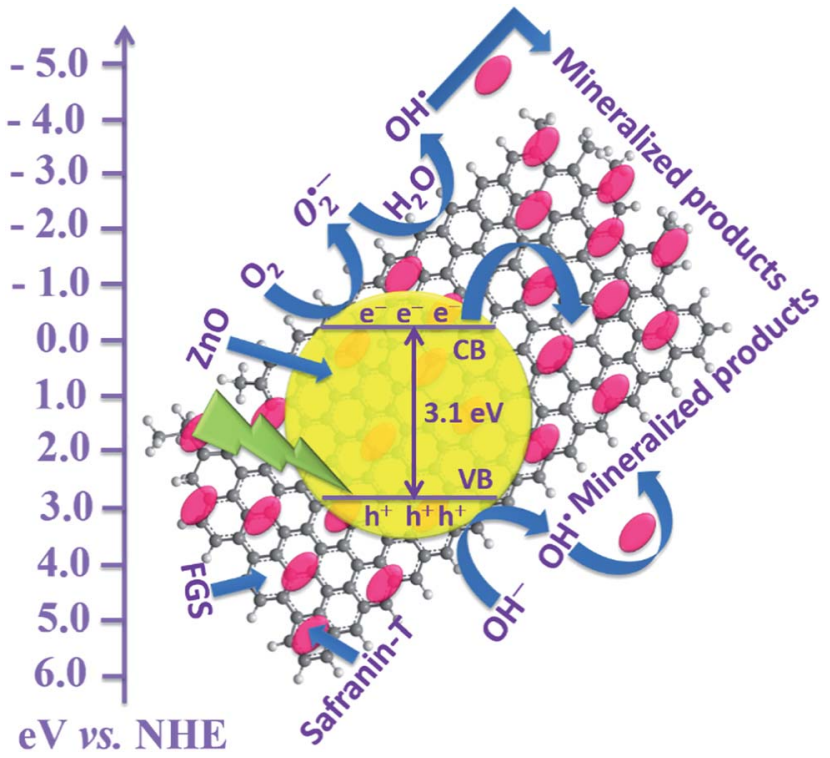

Fig. 6 Proposed photocatalytic mechanism for the degradation of safranin-T dye using FGS/ZnO NCs. ${ }^{24,27,29}$

$\left(\mathrm{h}^{+}\right)$, superoxide radical anions $\left(\mathrm{O}_{2}{ }^{-}\right)$and hydroxyl radicals $\left({ }^{\circ} \mathrm{OH}\right)$ are responsible for the mineralization of safranin-T dye into various eco-friendly intermediates. ${ }^{\mathbf{2 4 , 2 7}}$

In the present case, the real $\mathrm{VB}$ and $\mathrm{CB}$ of $\mathrm{ZnO}$ in $\mathrm{FGS} / \mathrm{ZnO}$ and bare $\mathrm{ZnO}$ have been estimated theoretically according to Mulliken electronegativity theory. ${ }^{45}$

$$
\begin{gathered}
E_{\mathrm{VB}}=X-4.5+0.5 E_{\mathrm{g}} \\
E_{\mathrm{CB}}=E_{\mathrm{VB}}-E_{\mathrm{g}}
\end{gathered}
$$

here, $E_{\mathrm{VB}}, E_{\mathrm{CB}}$ and $E_{\mathrm{g}}$ correspond to the valence band, conduction band and band gap of the individual semiconductor. $X$ is the absolute electronegativity of the corresponding semiconductor. The VB and $\mathrm{CB}$ of bare $\mathrm{ZnO}$ and various FGS/ZnO NCs were calculated and presented in Table $\mathrm{S} 2 . \dagger$ The observed VB and CB band positions indicate that $0.09 \mathrm{wt} \% \mathrm{FGS} / \mathrm{ZnO}$ NCs possess a lower conduction band and lower valence band than other FGS/ZnO NCs as well as bare $\mathrm{ZnO}$, which facilitates fast electron transport to the surface and enhances photocatalytic activity towards the photodegradation of safranin-T dye. The increased photocatalytic activity of FGS/ZnO NCs could be due to the following factors: ${ }^{27}$ (i) the enhanced safranin-T dye adsorption on the surface of FGS via $\pi-\pi$ interactions which could be due to the optimum amount of FGS in the FGS/ZnO NCs (Fig. S2 $\dagger$ ), and (ii) FGS being able to act as an efficient support for the strong adherence of ZnO NPs, this combination can help effective separation of the photogenerated excitons as well as effective transportation of the charge carriers towards the surface of the photocatalyst for efficient mineralization of the safranin-T dye (Fig. 4).

ZnO NPs are known to produce reactive oxygen species (ROS) by interacting with aqueous media. ${ }^{46}$ In the present study, the generation of hydroxyl radicals over the surface of the photocatalysts during the photocatalytic study was confirmed using 
terephthalic acid as a probe molecule. ${ }^{45,56}$ The intensity of the PL emission was monitored at a $\lambda_{\max }$ of $425 \mathrm{~nm}$ corresponding to the formation of the adduct i.e. 2-hydroxy terephthalic acid (TAOH) formed via the reaction between terephthalic acid and $\mathrm{OH}^{\bullet}$ generated during the interaction of pristine $\mathrm{ZnO}$ and FGS/ ZnO NCs in safranin-T dye solution (Fig. 7a).

The order of PL emission peak intensity was found to be 0.09 wt $\%$ FGS/ZnO NCs $>0.10$ wt $\%$ FGS/ZnO NCs $>0.05$ wt $\%$ FGS/ZnO NCs > pristine $\mathrm{ZnO}>0.50 \mathrm{wt} \% \mathrm{FGS} / \mathrm{ZnO}$ NCs $>$ $5.00 \mathrm{wt} \% \mathrm{FGS} / \mathrm{ZnO}$ NCs. The observed order coincides with the PL intensities of pristine ZnO and FGS/ZnO NCs (Fig. 4). The observed results indicate that the $0.09 \mathrm{wt} \% \mathrm{FGS} / \mathrm{ZnO}$ NCs can produce a higher amount of $\mathrm{OH}^{*}$ than other photocatalysts, which supports the higher photocatalytic activity of $0.09 \mathrm{wt} \%$ FGS/ZnO NCs compared to that of other photocatalysts. ${ }^{45,56}$ In order to determine the role of ROS generation towards the photodegradation of safranin- $T$ dye, we have designed an
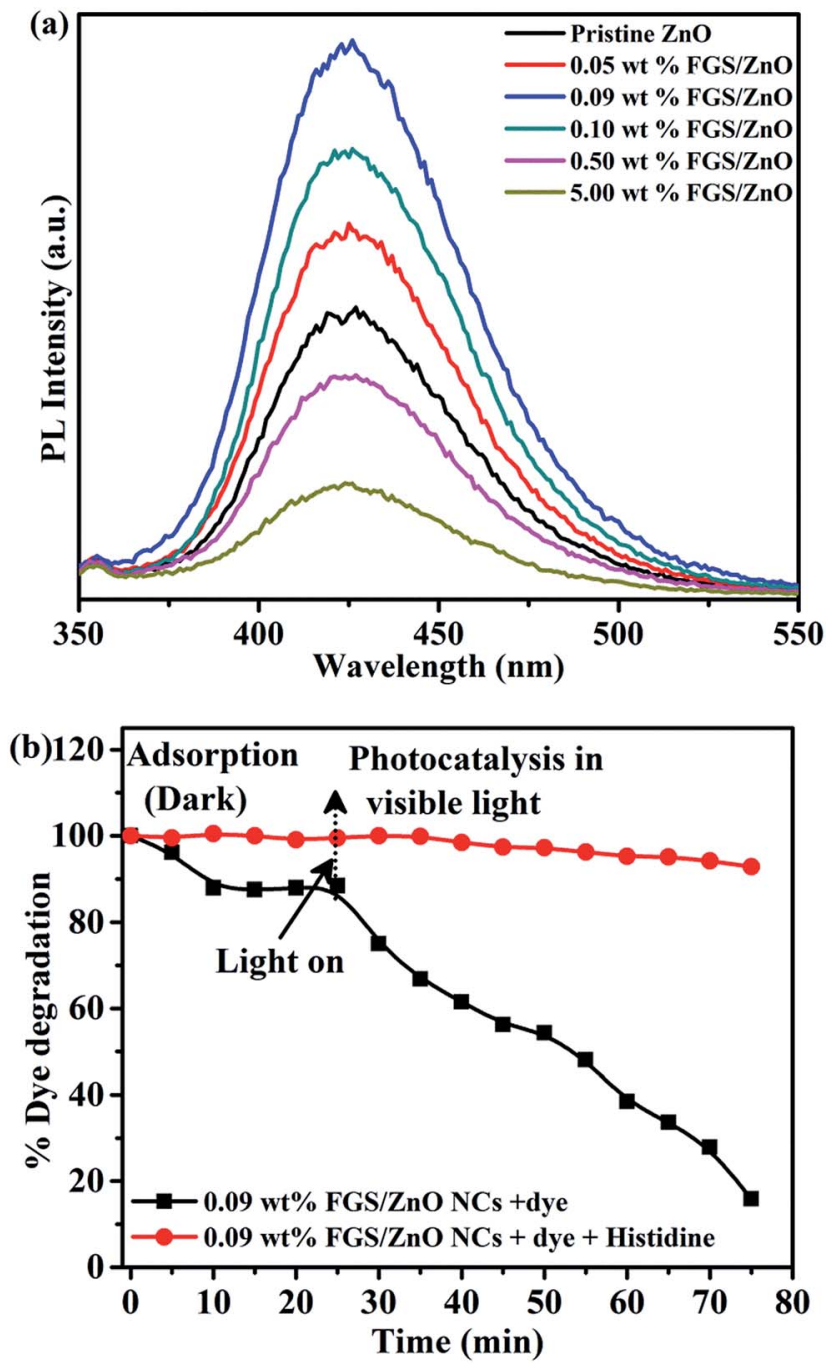

Fig. 7 (a) Determination of hydroxyl radical formation on the surface of various photocatalysts under visible light irradiation using photoluminescence spectroscopy $\left(\lambda_{\text {exc }}=315 \mathrm{~nm}\right)$ and $(b)$ photodegradation of safranin-T using $0.09 \mathrm{wt} \% \mathrm{FGS} / \mathrm{ZnO} \mathrm{NCs}$ in the presence of histidine as a scavenger. experiment to scavenge the generated ROS during the photodegradation of safranin-T dye in an aqueous solution. Here, histidine was used as the scavenger, which is a well-known scavenging agent for $\mathrm{OH}^{*}$ and singlet oxygen. ${ }^{46,47}$ Prior to the use of histidine as a scavenger, we carried out a control experiment with $45 \mathrm{mg}$ of histidine and without any photocatalyst using visible light illumination to study the effect of histidine on the photodegradation of safranin-T. The observed results indicate negligible photodegradation of safranin- $T$ dye by histidine (Fig. 7b). After that, the same amount of histidine was introduced along with $20 \mathrm{mg}$ of $0.09 \mathrm{wt} \% \mathrm{FGS} / \mathrm{ZnO}$ NC photocatalyst in $100 \mathrm{~mL}$ of safranin-T dye solution with visible light illumination and the photodegradation of safranin-T dye was monitored using UV-visible spectroscopy. The photodegradation of safranin-T dye was strongly inhibited (about $20 \%$ photodegradation of safranin- $\mathrm{T}$ dye), indicating that histidine strongly scavenges the generated $\mathrm{OH}^{*}$ during photocatalysis. The observed results strongly support the idea that photodegradation of safranin-T dye was induced by ROS generated during photocatalysis. ${ }^{46,47}$

\section{Conclusion}

FGS/ZnO NCs with enhanced photocatalytic activity towards the photodegradation of safranin-T dye in aqueous solution have been successfully synthesized via an economical, facile and simple solution route followed by calcination under environmental conditions. The effect of FGS on dye adsorption as well as photodegradation was explored. The photocatalyst with 0.09 wt $\%$ FGS/ZnO NCs exhibits high photocatalytic activity compared to pristine $\mathrm{ZnO}$ as well as other FGS/ZnO NCs. The enhancement in photodegradation of safranin- $T$ dye with $0.09 \mathrm{wt} \% \mathrm{FGS} / \mathrm{ZnO}$ NC photocatalyst was attributed to the fact it had an optimum amount of FGS for effective adsorption of safranin-T dye on the surface of FGS as well as an effective separation of charge carriers for the mineralization of safranin$\mathrm{T}$ dye. The increased amount of FGS causes more oxygen defects on the surface of the photocatalyst, which causes reduced activity by the shielding effect phenomenon. The role of ROS generation during the photocatalytic degradation of safranin-T dye was verified by PL measurement that used terephthalic acid as a probe molecule as well as a scavenger test by histidine. The reported synthetic method can be extended to prepare other FGS/semiconductor NCs for various functional applications.

\section{Conflicts of interest}

There are no conflicts to declare.

\section{Acknowledgements}

Bhavani Prasad Naik thanks the Zhejiang University, Govt. of China for the support. The authors are thankful to the Zhejiang University and Indira Gandhi Delhi Technical University for Women for providing the facilities. Bhavani Prasad Naik and Swathi Verma also give thanks to the Indian Institute of Technology Kanpur for providing the instrumental facilities. This 
work has been carried out under the Science \& Engineering Research Board (SERB), grant No. EEQ/2016/000616 and the authors are thankful to the SERB for the financial support.

\section{Notes and references}

1 K. Woan, G. Pyrgiotakis and W. Sigmund, Adv. Mater., 2009, 21, 2233-2239.

2 N. Zhang, Y. Zhang and Y. J. Xu, Nanoscale, 2012, 4, 57925813.

3 Q. Xiang, J. Yu and M. Jaroniec, Chem. Soc. Rev., 2012, 41, 782-796.

4 L. Abramian and H. El-Rassy, Chem. Eng. J., 2009, 150, 403-410.

5 K. Golka, S. Kopps and Z. W. Myslak, Toxicol. Lett., 2004, 151, 203-210.

6 I. Baranowska, C. Pieszko, D. Raróg and A. Pielesz, J. Environ. Sci. Health, Part A: Environ. Sci. Eng., 2002, 37, 1841-1848.

7 W. Chu and S. M. Tsui, Water Res., 2002, 36, 3350-3358.

8 E. Gao, W. Wang, M. Shang and J. Xu, Phys. Chem. Chem. Phys., 2011, 13, 2887-2893.

9 Q. Xiang, J. Yu and M. Jaroniec, Nanoscale, 2011, 3, 36703678.

10 Q. Xiang and J. Yu, J. Phys. Chem. Lett., 2013, 4, 753-759.

11 Q. Xiang, B. Cheng and J. Yu, Angew. Chem., Int. Ed., 2015, 54, 11350-11366.

12 B. Li and H. Cao, J. Mater. Chem. A, 2011, 21, 3346-3349.

13 Y. Zhang, N. Zhang, Z. R. Tang and Y. J. Xu, Phys. Chem. Chem. Phys., 2012, 14, 9167-9175.

14 S. Kandula and P. Jeevanandam, RSC Adv., 2015, 5, 7615076159.

15 C. Chen, W. Ma and J. Zhao, Chem. Soc. Rev., 2010, 39, 42064219.

16 T. Xu, L. Zhang, H. Cheng and Y. Zhu, Appl. Catal., B, 2011, 101, 382-387.

17 Z. Xiong, L. L. Zhang, J. Ma and X. S. Zhao, Chem. Commun., 2010, 46, 6099-6101.

18 S. Kandula and P. Jeevanandam, J. Nanopart. Res., 2014, 16, 2452.

19 C. Tian, Q. Zhang, A. Wu, M. Jiang, Z. Liang, B. Jiang and H. Fu, Chem. Commun., 2012, 48, 2858-2860.

20 F. Xu, P. Zhang, A. Navrotsky, Z. Y. Yuan, T. Z. Ren, M. Halasa and B. L. Su, Chem. Mater., 2007, 19, 5680-5686.

21 E. C. Salas, Z. Sun, A. Lu and J. M. Tour, ACS Nano, 2010, 4, 4852-4856.

22 B. P. Nenavathu, A. V. R. Krishna Rao, A. Goyal, A. Kapoor and R. K. Dutta, Appl. Catal., A, 2013, 459, 106-113.

23 J. Wu, X. Shen, L. Jiang, K. Wang and K. Chen, Appl. Surf. Sci., 2010, 256, 2826-2830.

24 J. Xu, Y. Cui, Y. Han, M. Hao and X. Zhang, RSC Adv., 2016, 6, 96778-96784.

25 Y. Yang, L. Ren, C. Zhang, S. Huang and T. Liu, ACS Appl. Mater. Interfaces, 2011, 3, 2779-2785.

26 B. Li, T. Liu, Y. Wang and Z. Wang, J. Colloid Interface Sci., 2012, 377, 114-121.

27 Z. Chen, N. Zhang and Y. J. Xu, CrystEngComm, 2013, 15, 3022.

28 F. S. Omar, H. N. Ming, S. M. Hafiz and L. H. Ngee, Int. J. Photoenergy, 2014, 2014, 1-9.
29 X. Liu, L. Pan, Q. Zhao, T. Lv, G. Zhu, T. Chen, T. Lu, Z. Sun and C. Sun, Chem. Eng. J., 2012, 183, 238-243.

30 X. Li, Q. Wang, Y. Zhao, W. Wu, J. Chen and H. Meng, J. Colloid Interface Sci., 2013, 411, 69-75.

31 P. V. Kamat, J. Phys. Chem. Lett., 2010, 1, 520-527.

32 Y. Zhang, Y. Zhang, L. Song, Y. Su, Y. Guo, L. Wu and T. Zhang, RSC Adv., 2018, 8, 885-894.

33 Y. Li, L. Wang, J. Ge, J. Wang, Q. Li, W. Wan, B. Zhang, X. Liu and W. Xue, RSC Adv., 2016, 6, 106508-106515.

34 H. Tian, K. Shen, X. Hu, L. Qiao and W. Zheng, J. Alloys Compd., 2017, 691, 369-377.

35 H. Tian, C. Wan, W. Zheng, X. Hu, L. Qiao and X. Wang, RSC $A d v .$, 2016, 6, 84722-84729.

36 S. Verma and R. K. Dutta, J. Environ. Chem. Eng., 2017, 5, 4776-4787.

37 H. Yu, P. Xiao, J. Tian, F. Wang and J. Yu, ACS Appl. Mater. Interfaces, 2016, 8, 29470-29477.

38 Y. Xu, Y. Mo, J. Tian, P. Wang, H. Yu and J. Yu, Appl. Catal., B, 2016, 181, 810-817.

39 X. Yang, J. Qin, Y. Jiang, K. Chen, X. Yan, D. Zhang, R. Li and H. Tang, Appl. Catal., B, 2015, 166-167, 231-240.

40 X. Chen, H. Zhang, D. Zhang, Y. Miao and G. Li, Appl. Surf. Sci., 2018, 435, 468-475.

41 M. Cao, P. Wang, Y. Ao, C. Wang, J. Hou and J. Qian, Chem. Eng. J., 2015, 264, 113-124.

42 X. Yang, J. Qin, Y. Li, R. Zhang and H. Tang, J. Hazard. Mater., 2013, 261, 342-350.

43 X. Yang, J. Qin, Y. Jiang, R. Li, Y. Li and H. Tang, RSC Adv., 2014, 4, 18627-18636.

44 W. J. Hummers and O. Richarde, J. Am. Chem. Soc., 1958, 80, 1339.

45 S. Tonda, S. Kumar, S. Kandula and V. Shanker, J. Mater. Chem. A, 2014, 2, 6772.

46 R. K. Dutta, B. P. Nenavathu, M. K. Gangishetty and A. V. R. Reddy, Colloids Surf., B, 2012, 94, 143-150.

47 G. Appierot, A. Lipovsky, R. Dror, N. Perkas, Y. Nitzan, R. Lubart and A. Gedanken, Adv. Funct. Mater., 2009, 19, 842-852.

48 S. Kandula and P. Jeevanandam, RSC Adv., 2015, 5, 52955306.

49 S. Kandula and P. Jeevanandam, J. Alloys Compd., 2014, 615, 167-176.

50 G. Guerguerian, F. Elhordoy, C. J. Pereyra, R. E. Marotti, F. Martín, D. Leinen, J. R. Ramos-Barrado and E. A. Dalchiele, Nanotechnology, 2011, 22, 505401.

51 H. Li, C. Yao, L. Meng, H. Sun, J. Huang and Q. Gong, Electrochim. Acta, 2013, 108, 45-50.

52 S. Bhattacharyya and A. Gedanken, J. Phys. Chem. C, 2008, 112, 659-665.

53 V. Ischenko, S. Polarz, D. Grote, V. Stavarache, K. Fink and M. Driess, Adv. Funct. Mater., 2005, 15, 1945-1954.

54 B. Liu, A. Khare and E. S. Aydil, ACS Appl. Mater. Interfaces, 2011, 3, 4444-4450.

55 F. Sun, F. Tan, W. Wang, X. Qiao and X. Qiu, Mater. Res. Bull., 2012, 47, 3357-3361.

56 S. Wang, H. Qian, Y. Hu, W. Dai, Y. Zhong, J. Chen and X. Hu, Dalton Trans., 2013, 42, 1122-1128. 